\title{
Verletzungen der Wirbelsäule - viele Fragen sind noch offen!
}

$D_{i}$ Behandlung wirbelsäulenverletzter Patienten erfuhr in den letzten 20 Jahren wesentliche Veränderungen. Die Vorteile der operativen Stabilisierung, besonders bei sehr instabilen Verletzungen und Patienten mit Querschnittläsion erkennen inzwischen auch Verfechter konservativer Behandlungskonzepte an. Immer mehr setzt sich auch die AO-Klassifikation von Verletzungen der thorakolumbalen Wirbelsäule nach Magerl et al. durch und hat bereits jetzt wesentlich zu einem einheitlicheren Sprachgebrauch sowie vergleichbareren Nachuntersuchungsergebnissen geführt. Ebenso konnten inzwischen andere Kontroversen vergangener Tage durch eine Fortentwicklung von Operationstechniken und Implantaten sowie die Beobachtung größerer Patientenkollektive über längere Zeiträume entschieden werden. Als Beispiel sei die Frage des „richtigen“ Zugangs zur unteren HWS genannt. Die ventrale, interkoporelle Spondylodese darf heute als Standardverfahren bezeichnet werden.

Während also - besonders bei häufigen Verletzungsformen - therapeutische Konzepte unterschiedlicher Chirurgen einheitlicher ausfallen als früher, gilt es heute vor allem, die vorhandenen Mög- lichkeiten durch eine verbesserte klinische und röntgenologische Dokumentation kritisch zu überprüfen und dadurch die Qualität der Behandlungen weiter $z u$ verbessern. Nicht so sehr die Frage „Was ist machbar?", sondern „Was ist notwendig?" sollte - auch unter dem Gesichtspunkt sich ändernder Erwartungen der Patienten an eine „angemessene" Behandlung - unser Denken bestimmen und im Vordergrund stehen.

\section{Nicht die Frage "Was ist machbar", sondern "Was ist notwendig" sollte unser Denken bestimmen.}

Voraussetzungen für überzeugendere Antworten sind zunächst genauere Definitionen von Defiziten, die im Augenblick noch nicht erarbeitet sind.

So kann es in den beiden Themenheften „Die verletzte Wirbelsäule Teil 1 und Teil 2 " nicht darum gehen, einen vollständigen Überblick über den gegenwärtigen Stand der Dinge zu geben.Vielmehr war es u.a. unsere Absicht, „Nischen“ aufzuspüren, Teilbereiche auf dem weiten Feld der traumatisierten Wirbelsäule, auf denen noch große Unklarheit besteht. Auch für seltenere Läsionen benötigen wir allgemein anerkannte Richtlinien.
Unter diesem Aspekt sind die beiden ersten Beiträge von S. Schären und $B$. Jeanneret sowie C. Josten zu sehen. Besonders für dislozierte Atlasfrakturen, aber auch für die traumatische Spondylolisthese C2 fehlen - nicht zuletzt durch die kleinen Patientenzahlen - eindeutige Empfehlungen. Das gilt auch für Verletzungen der Brust- und Lendenwirbelsäule bei Kindern.

A. Kathrein et al. stellen den aktuellen Stand des Wissens, untermauert durch eigene Nachuntersuchungen, dar.

„Qualitätsmanagement, und „Evidence based Medicine" - diese Schlagwörter sind in aller Munde (siehe „Der Orthopäde"Heft 3/99). Neue operationstechnische und apparative Möglichkeiten verführen manchen Operateur und beeinflussen neben regionalen Unterschieden die Indikationsstellung. Besonders aufwendige Operationsverfahren mit erhöhtem Risiko und nicht selten größerer Morbidität der Patienten bedürfen einer sorgfältigen Nachkontrolle und Rechtfertigung. T. Kossmann et al. stellen sich dieser Aufgabe für die kombinierte Operation bei Frakturen des thorakolumbalen Übergangs. 
Ein inzwischen schon sehr bewährtes „Instrument“ in diesem Sinne stellt die „Arbeitsgemeinschaft Wirbelsäule“ der Deutschen Gesellschaft für Unfallchirurgie dar. Prospektive, multizentrische Studien weisen naturgemä $\beta$ ein größeres Gewicht auf, als die häufig retrospektiven Untersuchungen aus einer einzigen Klinik. Dieses „Instrument" gilt es auch in Zukunft weiter zu nutzen. M. Hofmeister und V. Bühren unterstützen ihr Therapiekonzept für Verletzungen der unteren HWS mit zahlreichen Daten aus einer Sammelstudie $\operatorname{der} A G$ Wirbelsäule.

Der Beschleunigungsverletzung der HWS kommt große sozioökonomische Bedeutung zu. Auch und gerade hier spielen Meinungen und „Mythen in der Medizin" [Krämer et al. (1999): Dokumentation und Qualitätsmanagement - ein Gebot der Zeit. Orthopäde 28:18919o] immer noch eine viel größere Rolle als Fakten und nachvollziehbare Erkenntnisse. Von unfallchirurgischer Seite findet man aus den letzten Jahren kaum Beiträge zu diesem Problemkreis. M. Richter et al. nutzen den in Deutschland einmaligen, u.a. an der Unfallstelle erhobenen Datenbestand der Verkehrsunfallforschung an der Medizinischen Hochschule Hannover und berichten über Zusammenhänge zwischen klinischem Verlauf nach einer Distorsion der HWS und technische Angaben zur Unfallstelle, Auswertungen der Pkw-Deformierung und über medizinische Details zu Art und Schwere von anfänglichen Personenschäden. Danach müssen manche frühere Vorstellungen überdacht werden.

Den Abschluß bildet eine Arbeit von $H$. Graichen und R. Putz über neuere anatomische und funktionelle Aspekte der Brust- und Lendenwirbelsäule. Die Autoren legten absprachegemä $\beta$ besonderes Gewicht auf solche Details, die bei den Zugängen zur Wirbelsäule oder einer Instrumentierung von Wirbeln eine Rolle spielen; sie leisten damit einen wertvollen Beitrag zur Differentialindikation und „operativen Sicherheit“.
Danken möchte ich allen Autoren für ihre sofort geäußerte Bereitschaft zur Mitwirkung an diesem Heft sowie dafür, daß sie den sehr engen zeitlichen Rahmen akzeptiert und auch eingehalten haben.

\section{Manín}

M. Blauth 